Supplement of Atmos. Chem. Phys. Discuss., 14, 21975-22043, 2014

http://www.atmos-chem-phys-discuss.net/14/21975/2014/

doi:10.5194/acpd-14-21975-2014-supplement

(C) Author(s) 2014. CC Attribution 3.0 License.

(c) (i)

\title{
Aerosol-cloud interactions studied with the chemistry-climate model EMAC
}

D. Y. Chang et al.

Correspondence to: D. Y. Chang (dongyeong.chang@mpic.de) 
Table S1. Hygroscopicity of aerosol components characterized by the number of ions the salt dissociates into in water $(\mu)$, osmotic coefficient $(\phi)$, mass fraction of soluble material $(\epsilon)$, density $\left(\rho_{a}\right)$ and molecular weight $\left(M_{a}\right)$ of aerosol. 


\begin{tabular}{lccccc||c|c}
\hline Component $(j)$ & $\mu_{j}$ & $\phi_{j}$ & $\epsilon_{j}$ & $\rho_{j}$ & $M_{j}$ & $B_{j}$ & $\kappa_{j}$ \\
\hline \hline Ammonium sulfate & 3.0 & 0.7 & 1.0 & 1.77 & 132.17 & 0.51 & 0.61 \\
Sea salt & 2.0 & 1.0 & 1.0 & 2.17 & 58.44 & 1.16 & 1.28 \\
Soil dust & 2.0 & 0.45 & 0.13 & 2.65 & 40.08 & 0.14 & 0.03 \\
Organic carbon & 1.0 & 1.0 & 0.047 & 2.0 & 12.11 & 0.14 & 0.1 \\
Black carbon & 1.0 & 1.0 & $1.7 \mathrm{e}-07$ & 2.0 & 12.11 & $0.5 \mathrm{e}-7$ & 0.0 \\
\hline
\end{tabular}

For the multi-compound bulk aerosols (i.e., DU, BC, and OC) empirical parameters or estimations based on experiments are applied to the calculation of $B_{j}$; the osmotic coefficient $\left(\phi_{D U}\right)$ is based on east Asian dust observed by Nishikawa et al. (1991) and Nishikawa (1993). To simplify the calculation of water uptake, the fixed parameters of $\phi_{j}$ and $B_{j}$ are applied, which could be varied depending on the solution. The density $\left(\rho_{D U}\right)$ and molecular weight $\left(M_{D U}\right)$ of soil dust are based on the chemical composition of $\mathrm{CaSO}_{4}$ in the aerosol submodel GMXe. 


\subsection{Taylor Diagram}

Taylor diagrams based on Taylor $(2001,2005)$ summarizes the resemblance between two patterns (e.g., simulations and observation) with statistical values (i.e., the centered root mean squared (RMS) difference, the spatial pattern correlation, and the standard deviation). A Taylor diagram can be described with the following formula (Taylor, 2001):

$E^{\prime 2}=\sigma_{x}^{2}+\sigma_{r}^{2}-2 \sigma_{x} \sigma_{r} R$,

where $R$ is the correlation coefficient, $E^{\prime}$ is the centered $R M S$ difference between the model results and the observations (or reference data), and $\sigma_{x}$ and $\sigma_{r}$ are the standard deviations of the model results and the observations (or reference data), are given by;

$R=\frac{\frac{1}{N} \sum_{n=1}^{N}\left(x_{n}-\bar{x}\right)\left(r_{n}-\bar{r}\right)}{\sigma_{x} \sigma_{r}}$

$E^{\prime 2}=\frac{1}{N} \sum_{n=1}^{N}\left[\left(x_{n}-\bar{x}\right)-\left(r_{n}-\bar{r}\right)\right]^{2}$

$\sigma_{x}^{2}=\frac{1}{N} \sum_{n=1}^{N}\left[\left(x_{n}-\bar{x}\right)\right]^{2}, \quad \sigma_{r}^{2}=\frac{1}{N} \sum_{n=1}^{N}\left[\left(r_{n}-\bar{r}\right)\right]^{2}$

To plot different variables (i.e., the relevant cloud and climate parameters) on the same diagram, the simulated standard deviation and centered RMS error are normalized by the corresponding observed standard deviation $\left(\sigma_{r}\right)$. Note that the correlation coefficient $(\mathrm{R})$ is not changed by a normalization. The normalized statistical variables are calculated with the following equation;

$\hat{E}^{\prime}=\frac{E^{\prime}}{\sigma_{r}}, \hat{\sigma_{x}}=\frac{\sigma_{x}}{\sigma_{r}}, \hat{\sigma_{r}}=1$ 


\subsection{Skill score}

To evaluate the performance of the EMAC model, a relative skill score is calculated with by the following formula (Taylor, 2001)

$$
S=\frac{4(1+R)}{\left(\hat{\sigma_{x}}+\frac{1}{\sigma_{x}}\right)^{2}\left(1+R_{0}\right)},
$$

where $R_{0}$ is the maximum correlation coefficient (0.9976) in this study. This model skill score is determined by the pattern variability and correlation with observations. If the simulated standard deviation is close to the observed standard deviation (i.e., $\hat{\sigma_{x}}$ approaches 1 ) and the correlation coefficient increases (i.e., $\mathrm{R}$ is close to $R_{0}$ ), the model skill score (S) approaches 1 (most skillful). Note that the RMS error may not necessarily contribute to a high skill score. Further detailed information can be found in Taylor (2001). 
Table S2. Continental and marine means and corresponding statistic variables (i.e., the standard deviation $(\sigma)^{*}$, spatial pattern correlation coefficient $(R)$, centered root mean square $\left(E^{\prime}\right)^{*}$ difference) used in the spatial pattern comparisons (Fig. ??) between the model simulations and observations, and their skill scores $(S)$ (Table ??**). 


\begin{tabular}{|c|c|c|c|c|c|c|c|c|c|c|}
\hline & \multicolumn{5}{|c|}{ Continental } & \multicolumn{5}{|c|}{ Marine } \\
\hline TCC ( - ) & Mean & $\sigma$ & $R$ & $E^{\prime}$ & $S$ & Mean & $\sigma$ & $R$ & $E^{\prime}$ & $S$ \\
\hline 1-RH-REF & 0.532 & 0.204 & 0.903 & 0.088 & 0.9513 & 0.715 & 0.158 & 0.865 & 0.079 & 0.9205 \\
\hline 2-ST-REF & 0.482 & 0.195 & 0.855 & 0.105 & 0.9287 & 0.539 & 0.198 & 0.789 & 0.122 & 0.7975 \\
\hline 3-RH-STN & 0.548 & 0.200 & 0.898 & 0.090 & 0.9497 & 0.722 & 0.154 & 0.868 & 0.077 & 0.9263 \\
\hline 4-ST-STN & 0.533 & 0.219 & 0.900 & 0.095 & 0.9401 & 0.618 & 0.169 & 0.786 & 0.105 & 0.8625 \\
\hline 5-RH-HYB & 0.539 & 0.202 & 0.903 & 0.090 & 0.9518 & 0.717 & 0.157 & 0.863 & 0.077 & 0.9207 \\
\hline 6-ST-HYB & 0.493 & 0.195 & 0.876 & 0.095 & 0.9392 & 0.563 & 0.191 & 0.817 & 0.105 & 0.8281 \\
\hline Obs. (MODIS) & 0.520 & 0.196 & & & & 0.7155 & 0.140 & & & \\
\hline 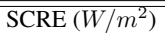 & Mean & $\sigma$ & 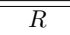 & 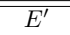 & $\overline{S S}$ & Mean & $\overline{\bar{\sigma} \sigma}$ & 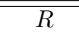 & 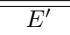 & $\overline{S S}$ \\
\hline 1-RH-REF & -35.76 & 24.36 & 0.897 & 11.03 & 0.9025 & -64.79 & 20.59 & 0.761 & 13.51 & 0.8599 \\
\hline 2-ST-REF & -24.36 & 14.68 & 0.854 & 10.27 & 0.8592 & -37.18 & 19.24 & 0.764 & 12.74 & 0.8761 \\
\hline 3-RH-STN & -45.40 & 30.76 & 0.906 & 15.51 & 0.7778 & -76.74 & 22.94 & 0.736 & 15.54 & 0.8106 \\
\hline 4-ST-STN & -43.36 & 30.76 & 0.863 & 17.08 & 0.7603 & -55.51 & 22.26 & 0.741 & 14.99 & 0.8248 \\
\hline 5-RH-HYB & -38.23 & 25.38 & 0.909 & 15.51 & 0.8901 & -68.99 & 21.90 & 0.762 & 15.54 & 0.8410 \\
\hline 6-ST-HYB & -27.63 & 16.54 & 0.867 & 17.08 & 0.9111 & -43.29 & 21.59 & 0.768 & 14.99 & 0.8481 \\
\hline Obs. (CERES) & -33.00 & 19.42 & & & & -52.84 & 17.59 & & & \\
\hline$\overline{\mathrm{LCCRE}}\left(W / m^{2}\right)$ & Mean & $\sigma$ & 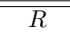 & 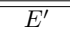 & $\overline{S S}$ & Mean & 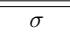 & 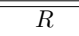 & 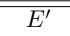 & $\overline{S S}$ \\
\hline 1-RH-REF & 26.07 & 11.63 & 0.894 & 5.336 & 0.9479 & 29.48 & 13.24 & 0.868 & 6.595 & 0.9005 \\
\hline 2-ST-REF & 24.98 & 13.07 & 0.892 & 5.912 & 0.9319 & 23.97 & 11.87 & 0.826 & 6.777 & 0.9074 \\
\hline 3-RH-STN & 24.77 & 9.876 & 0.811 & 6.752 & 0.8858 & 30.19 & 13.19 & 0.864 & 6.655 & 0.8998 \\
\hline 4-ST-STN & 22.51 & 9.243 & 0.839 & 6.275 & 0.8778 & 26.66 & 11.89 & 0.865 & 5.989 & 0.9266 \\
\hline 5-RH-HYB & 25.96 & 11.11 & 0.881 & 6.752 & 0.9405 & 29.59 & 13.12 & 0.868 & 6.655 & 0.9032 \\
\hline 6-ST-HYB & 25.02 & 12.87 & 0.899 & 6.275 & 0.9391 & 24.62 & 11.88 & 0.830 & 5.989 & 0.9091 \\
\hline Obs. (CERES) & 24.17 & 11.51 & & & & 27.09 & 10.89 & & & \\
\hline Ptot (mm/day) & Mean & $\overline{\sigma \sigma}$ & R & 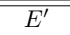 & $\overline{S S}$ & Mean & $\overline{\sigma \sigma}$ & R & $\bar{E}^{\prime}$ & $\overline{S S}$ \\
\hline 1-RH-REF & 2.142 & 1.975 & 0.827 & 1.135 & 0.9117 & 3.348 & 2.665 & 0.833 & 1.529 & 0.7950 \\
\hline 2-ST-REF & 2.788 & 3.000 & 0.844 & 1.741 & 0.7434 & 3.039 & 2.394 & 0.825 & 1.361 & 0.8477 \\
\hline 3-RH-STN & 1.752 & 1.419 & 0.803 & 1.115 & 0.8378 & 3.441 & 2.681 & 0.814 & 1.600 & 0.7834 \\
\hline 4-ST-STN & 1.998 & 1.792 & 0.892 & 0.855 & 0.9453 & 3.321 & 2.516 & 0.8409 & 1.394 & 0.8304 \\
\hline 5-RH-HYB & 2.067 & 1.840 & 0.829 & 1.115 & 0.9155 & 3.366 & 2.656 & 0.830 & 1.600 & 0.7957 \\
\hline 6-ST-HYB & 2.720 & 2.876 & 0.863 & 0.855 & 0.7783 & 3.074 & 2.372 & 0.8279 & 1.394 & 0.8530 \\
\hline Obs. (GPCP) & 2.118 & 1.868 & & & & 2.904 & 1.817 & & & \\
\hline AOD ( - ) & Mean & $\sigma$ & $R$ & $E^{\prime}$ & $\overline{S S}$ & Mean & 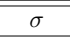 & 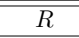 & 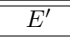 & $\overline{S S}$ \\
\hline 1-RH-REF & 0.217 & 0.167 & 0.784 & 0.108 & 0.8906 & 0.203 & 0.084 & 0.692 & 0.062 & 0.8219 \\
\hline 2-ST-REF & 0.202 & 0.156 & 0.817 & 0.095 & 0.9094 & 0.184 & 0.076 & 0.704 & 0.057 & 0.8489 \\
\hline 3-RH-STN & 0.236 & 0.174 & 0.759 & 0.117 & 0.8729 & 0.233 & 0.099 & 0.655 & 0.075 & 0.7429 \\
\hline 4-ST-STN & 0.227 & 0.179 & 0.803 & 0.108 & 0.8893 & 0.219 & 0.094 & 0.767 & 0.060 & 0.8183 \\
\hline 5-RH-HYB & 0.214 & 0.164 & 0.729 & 0.117 & 0.8646 & 0.215 & 0.087 & 0.635 & 0.075 & 0.7846 \\
\hline 6-ST-HYB & 0.200 & 0.155 & 0.769 & 0.108 & 0.8852 & 0.194 & 0.076 & 0.658 & 0.060 & 0.8260 \\
\hline Obs. (MODIS) & 0.219 & 0.159 & & & & 0.132 & 0.071 & & & \\
\hline
\end{tabular}

*The normalized standard deviation $\left(\hat{\sigma_{x}}=\frac{\sigma_{x}}{\sigma_{r}}\right)$ and CRMS difference $\left(\hat{E}^{\prime}=\frac{E^{\prime}}{\sigma_{r}}\right)$ by the corresponding observed standard deviation $\left(\sigma_{r}\right)$ are used in the Taylor diagrams (??). **Rating in Table ?? is based on skill 
score (S), with following ranges ; 5 (1.000-0.9500); 4 (0.9499-0.9000); 3 (0.8999-0.8500); 2 (0.84990.7500); 1 (0.7499-0.6000); 0 (under 0.6000) 
a) Sulfate

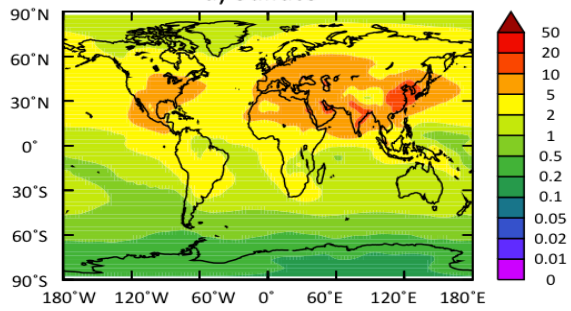

c) Sea salt

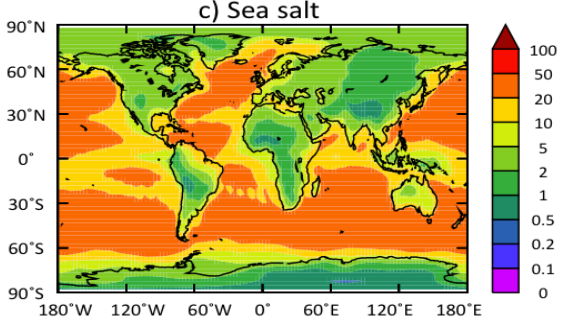

e) $\mathrm{BC}$

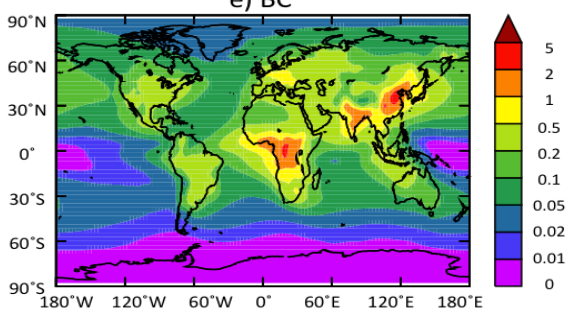

b) Ammonium

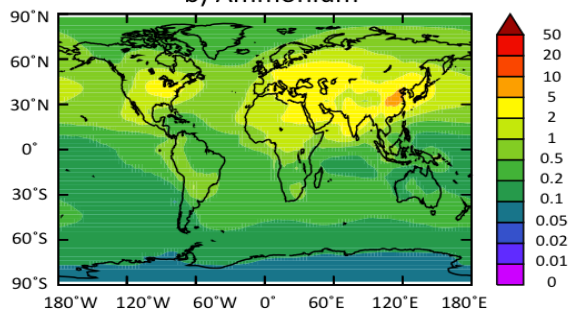

d) Mineral dust

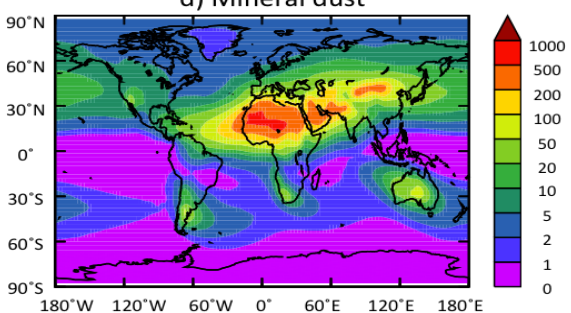

f) $\mathrm{OC}$

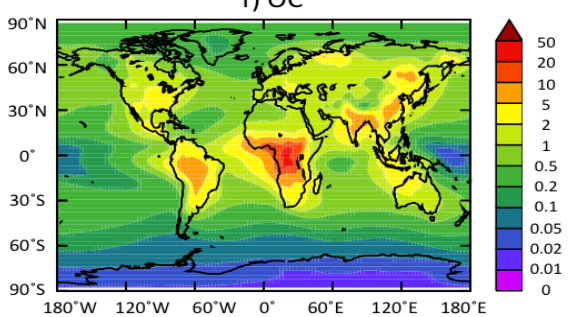

Fig. S1. Global distributions of the annual mean total aerosol column burden;(a) sulfate, (b) ammonium, (c) sea salt, (d) mineral dust, (e) BC, and (f) OC for ST-HYB, unit $\left(\mathrm{mg} / \mathrm{m}^{2}\right)$. 

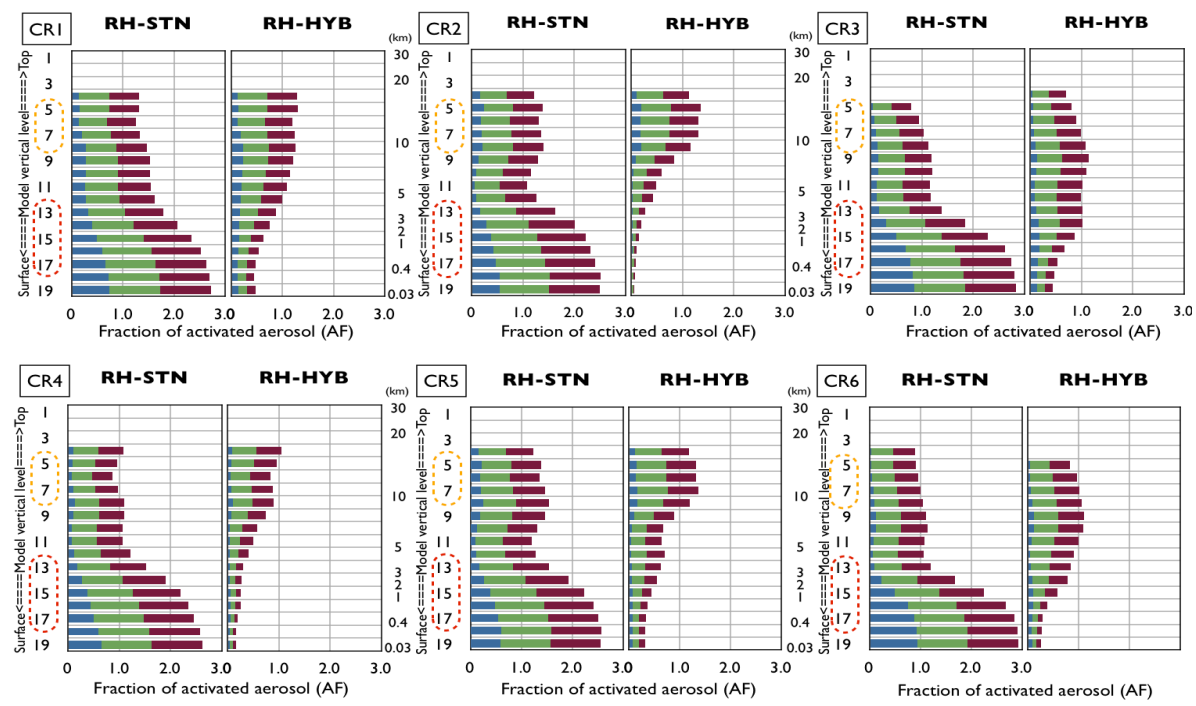

RH-STN RH-HYB

Tropopause $\quad$ PBL height

- Aitken

Accumulation

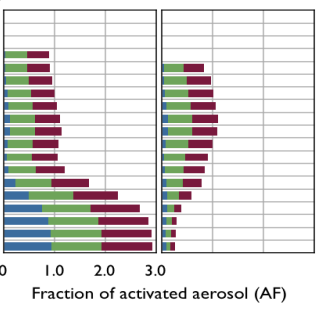

Coarse

Fig. S2. Vertical distributions of the activated aerosol fraction in the selected continental regions (from CR1 to CR6) for RH-STN and RH-HYB. 

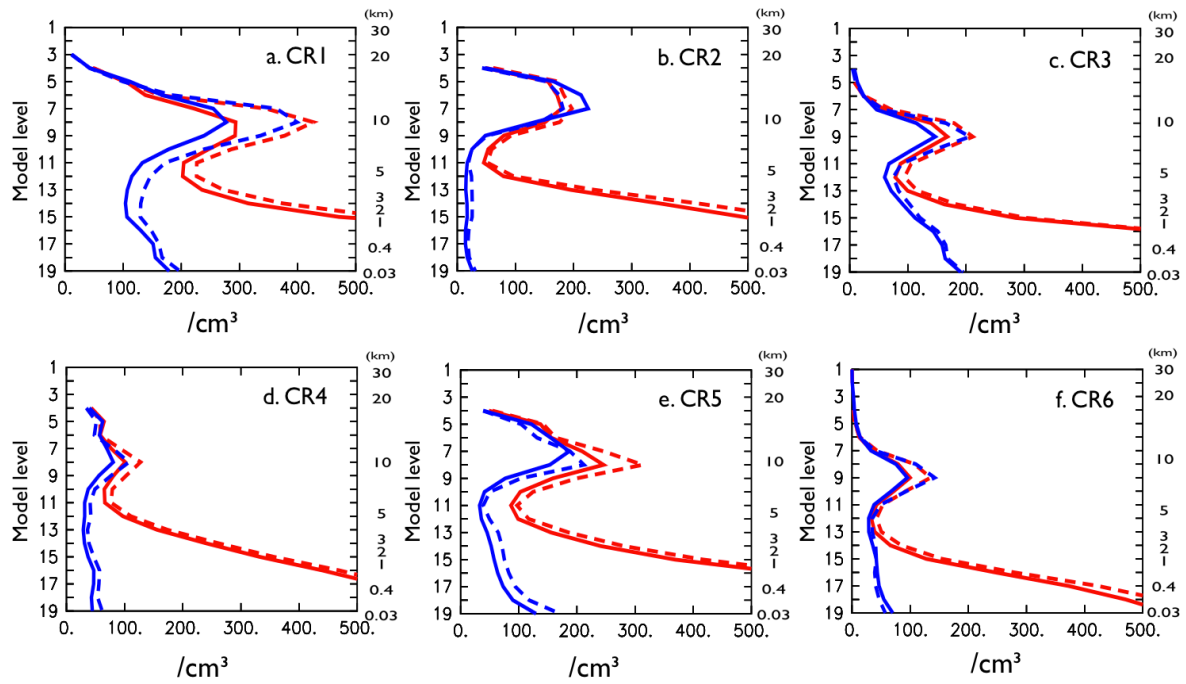

RH-STN (dash) ST-STN (solid) RH-HYB (dash) ST-HYB (solid)

Fig. S3. Vertical distributions of activated aerosol $(\mathrm{CCN})$ number concentration in the selected continental regions (CR) for RH-STN, ST-STN, RH-HYB, and ST-HYB. 

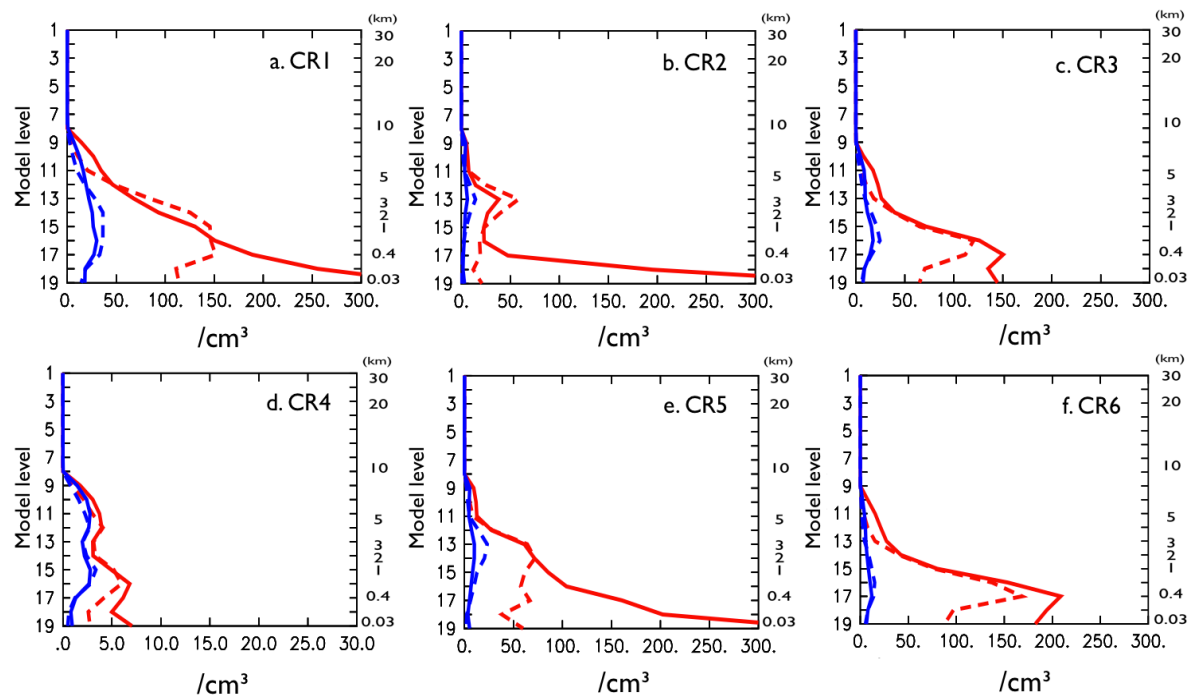

RH-STN (dash) ST-STN (solid) RH-HYB (dash) ST-HYB (solid)

Fig. S4. Vertical distributions of cloud droplet number concentration (CDNC) in the selected continental regions (CR) for RH-STN, ST-STN, RH-HYB, and ST-HYB. 

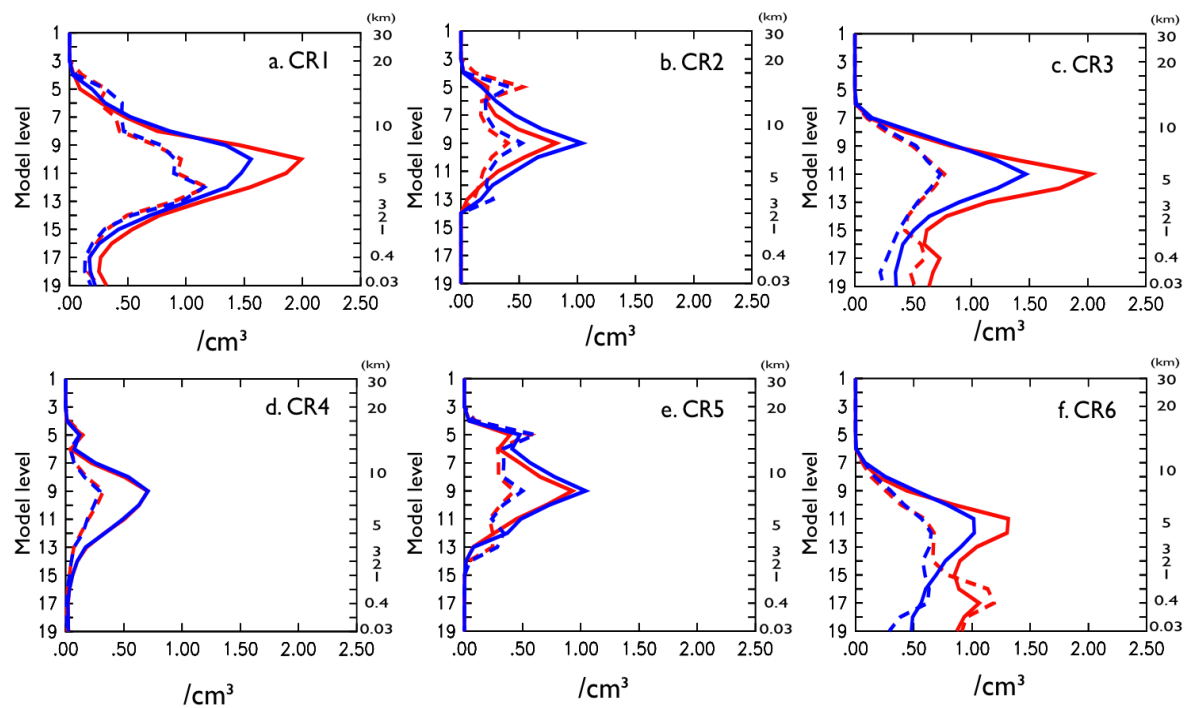

RH-STN (dash) ST-STN (solid) RH-HYB (dash) ST-HYB (solid)

Fig. S5. Vertical distributions of ice crystal number concentration (ICNC) in the selected continental regions (CR) for RH-STN, ST-STN, RH-HYB, and ST-HYB. 

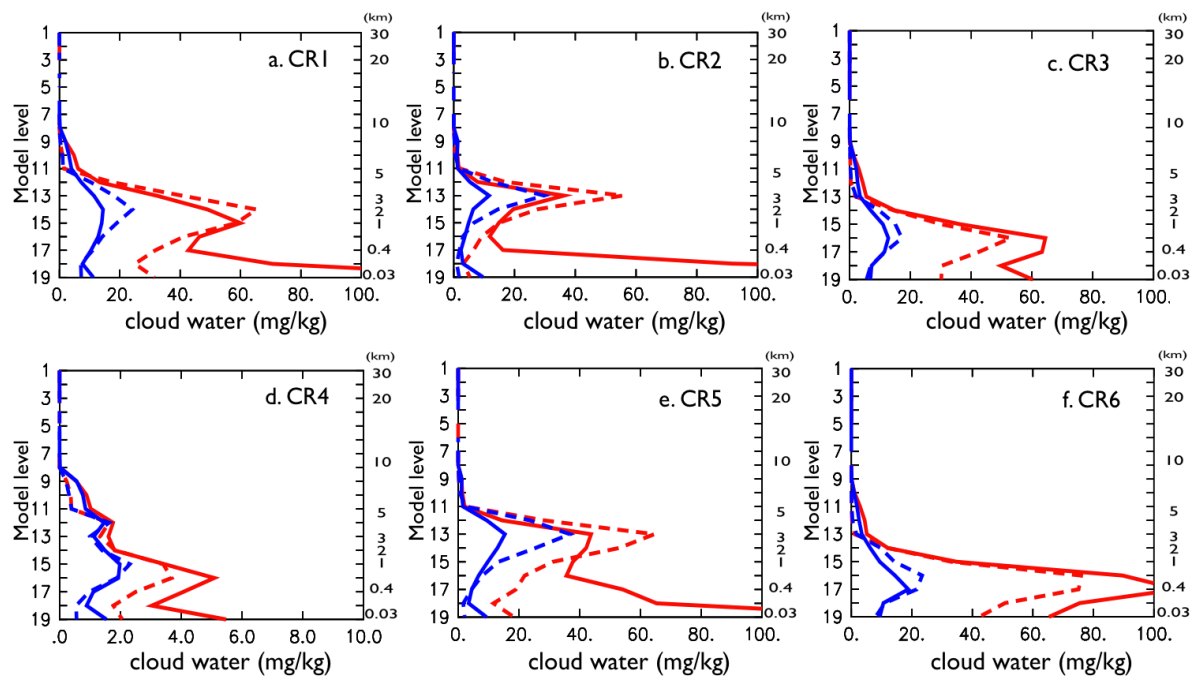

RH-STN (dash) ST-STN (solid) RH-HYB (dash) ST-HYB (solid)

Fig. S6. Vertical distributions of cloud liquid water in the selected continental regions (CR) for RH-STN, ST-STN, RH-HYB, and ST-HYB. 

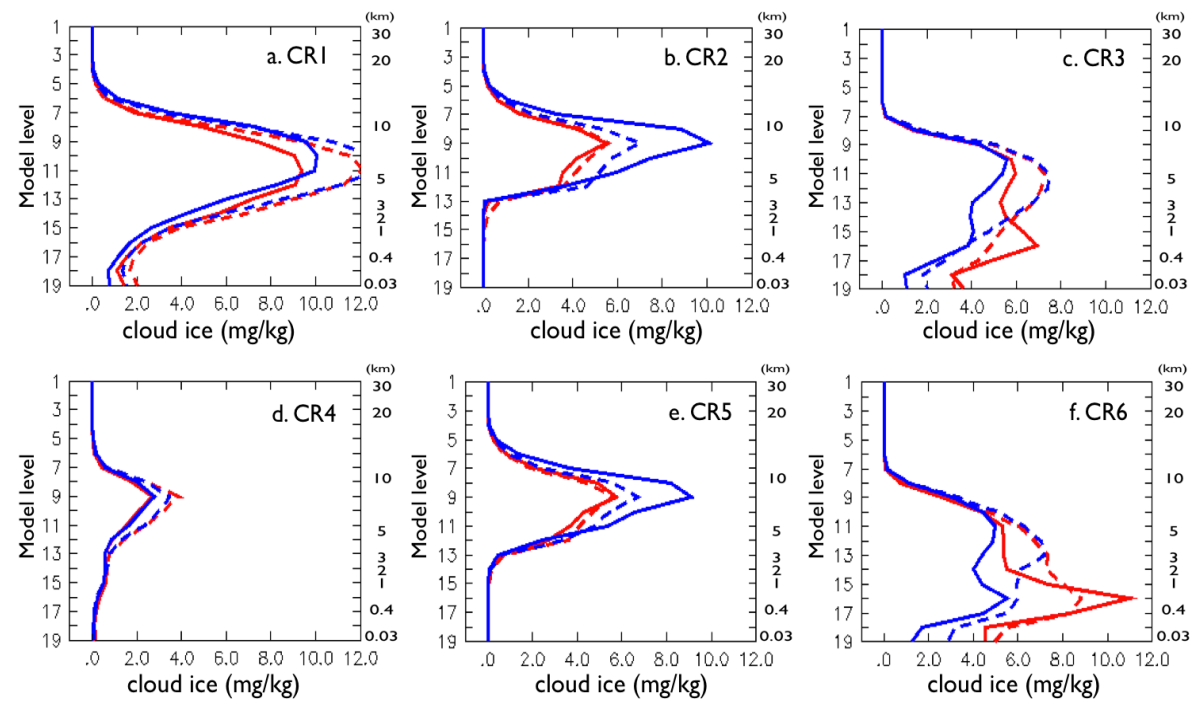

RH-STN (dash) ST-STN (solid) RH-HYB (dash) ST-HYB (solid)

Fig. S7. Vertical distributions of cloud ice in the selected continental regions (CR) for RH-STN, ST-STN, RH-HYB, and ST-HYB. 

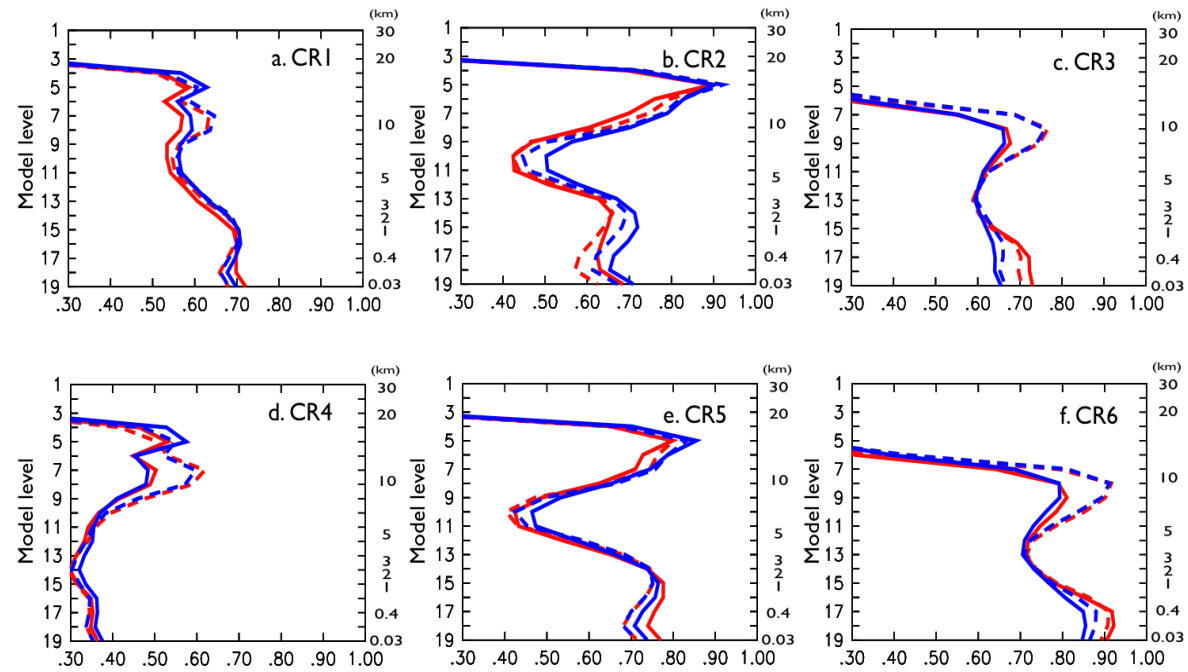

RH-STN (dash) ST-STN (solid) RH-HYB (dash) ST-HYB (solid)

Fig. S8. Vertical distributions of relative humidity in the selected continental regions (CR) for RH-STN, ST-STN, RH-HYB, and ST-HYB. 

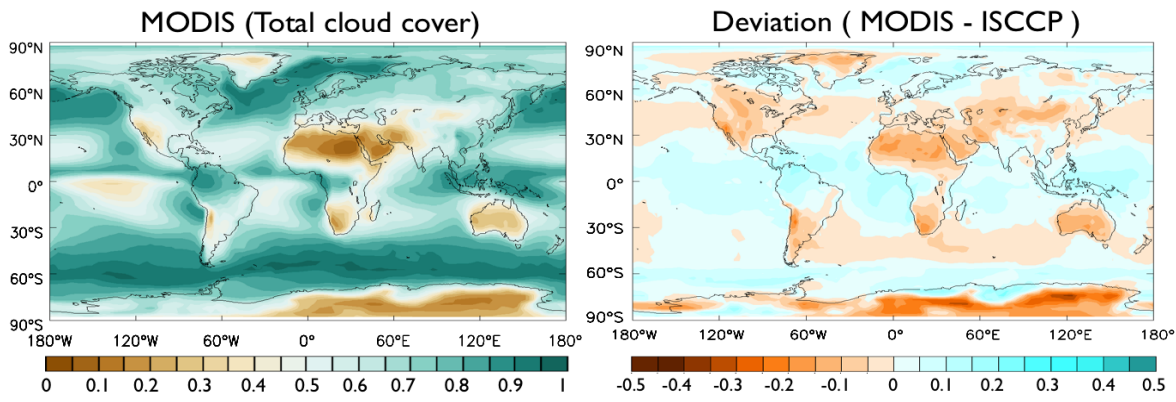

Fig. S9. Global distributions of total cloud cover fraction (TCC) derived by MODIS over a 10 year period and the difference of TCC between MODIS and ISCCP [MODIS-ISCCP]. 
a) RH-REF

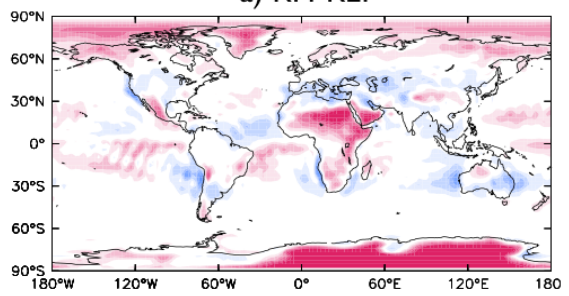

c) RH-STN

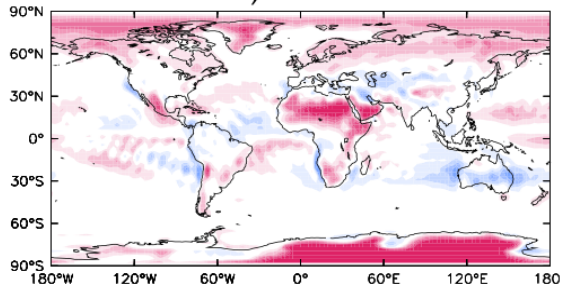

e) $\mathrm{RH}-\mathrm{HYB}$

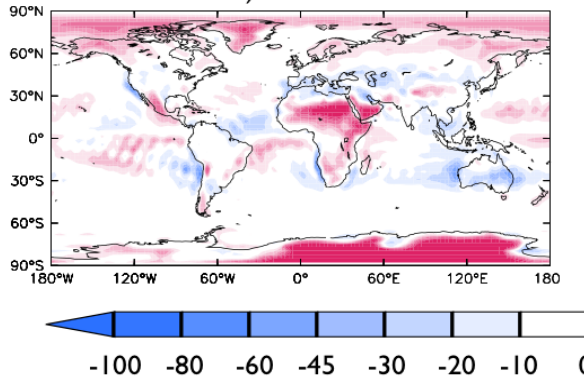

b) ST-REF

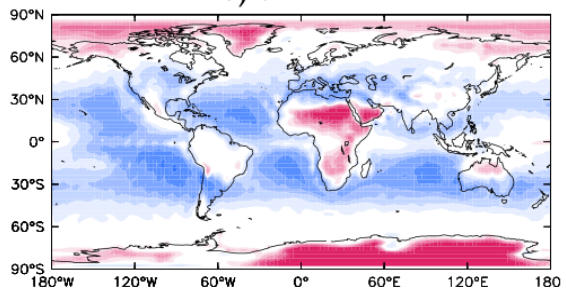

d) ST-STN

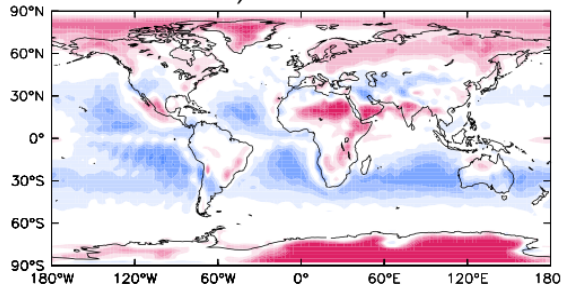

f) ST-HYB

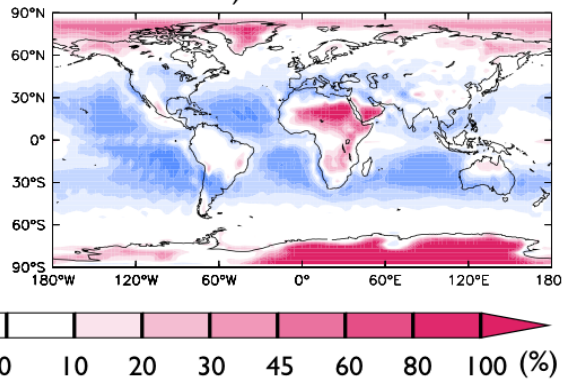

Fig. S10. Relative differences of TCC between simulations and observations (OBS) by MODIS over 10 years: Relative difference $(\%)=\frac{[\text { Model }-O B S]}{O B S} \times 100 \%$ 
b)

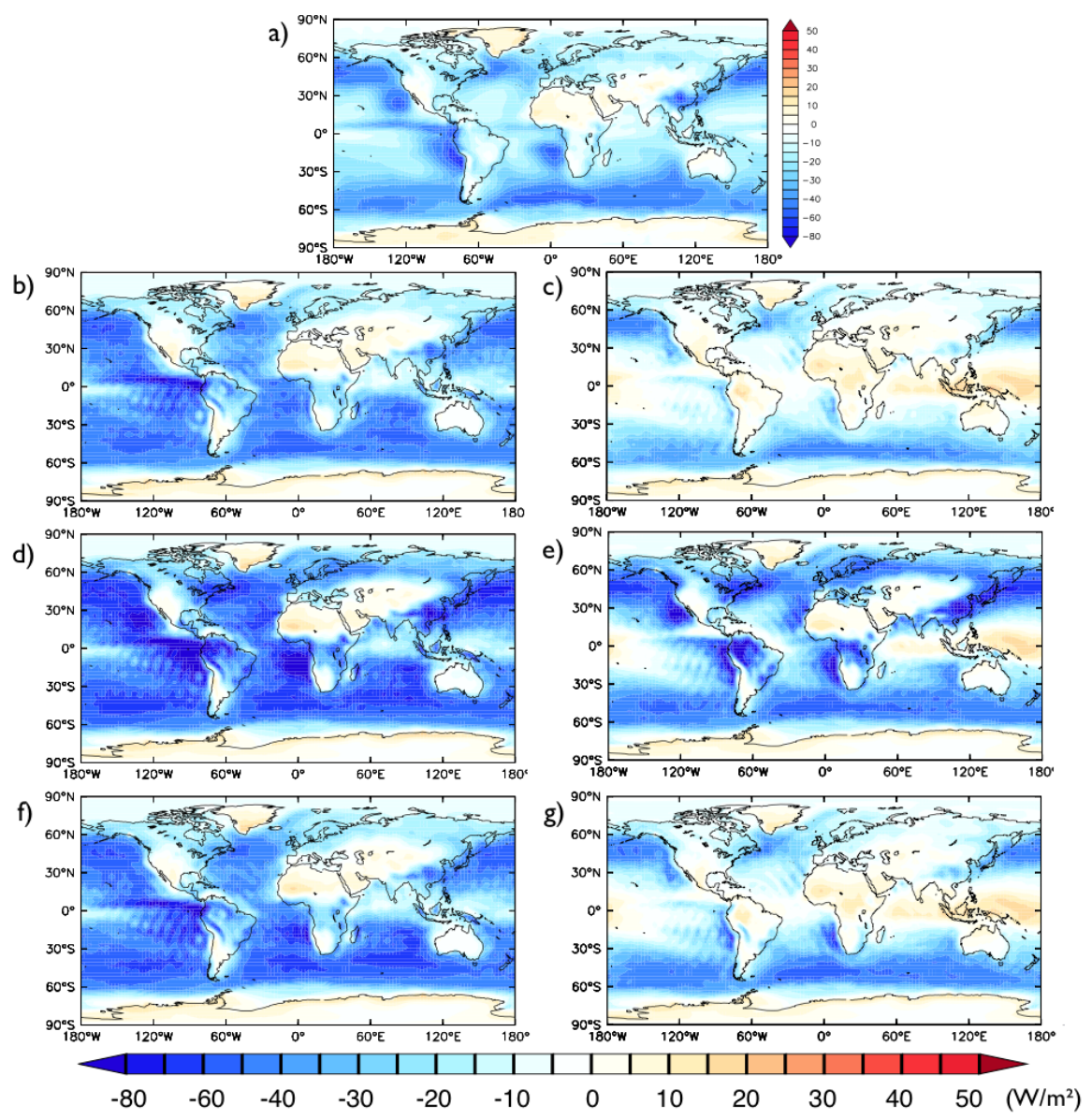

Fig. S11. Annual mean of NCRE at TOA for the simulations and the observational data: a) CERES EBAF (OBS), b) RH-REF, c) ST-REF, d) RH-STN, e) ST-STN, f) RH-HYB and g) ST-HYB 
a) RH-REF - OBS

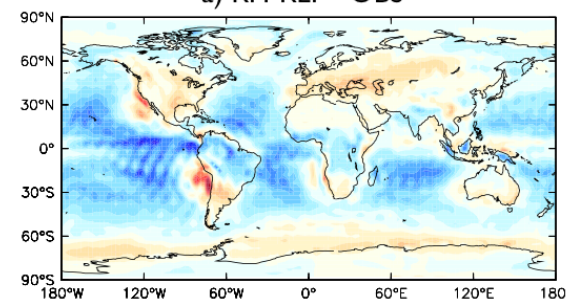

c) RH-STN - OBS

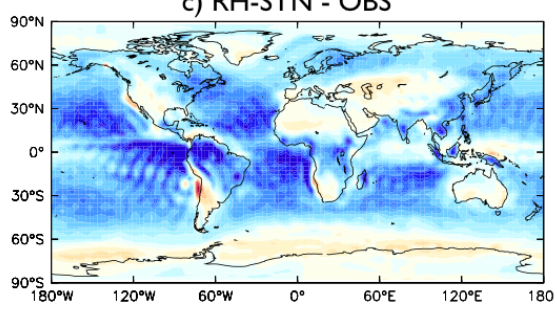

e) RH-HYB - OBS

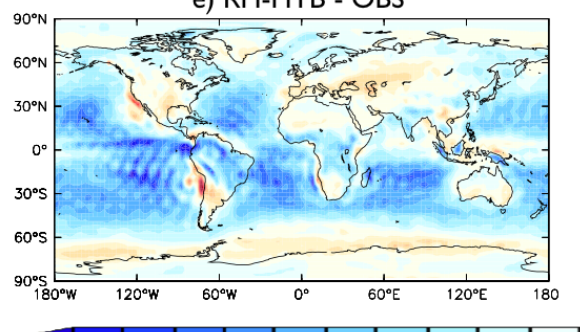

b) ST-REF - OBS

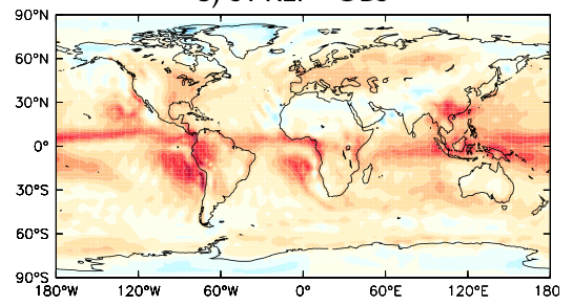

d) ST-STN - OBS

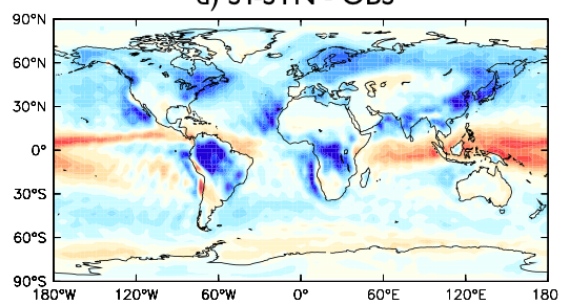

f) ST-HYB - OBS

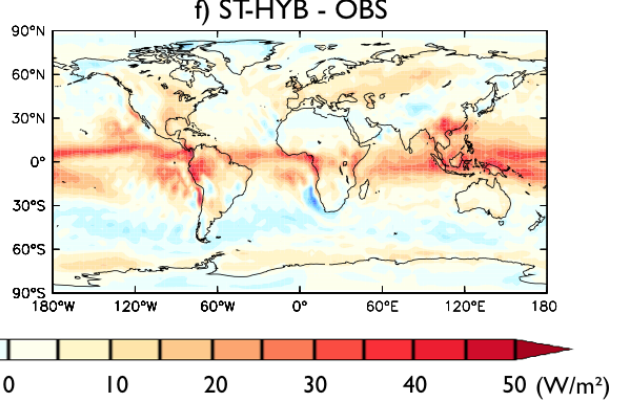

Fig. S12. Deviations of annual mean NCRE at TOA between the simulations and the observational data (CERES EBAF); a) RH-REF, b) ST-REF, c) RH-STN, d) ST-STN, e) RH-HYB, f) ST-HYB 